\title{
Múltiples xantomas en un paciente con síndrome de Alagille
}

\section{Multiple xanthomas in a patient with Alagille syndrome}

María Candela Viada Peláez ${ }^{1}$, María Marta Buján², María del Valle Centeno ${ }^{3}$ y Andrea Bettina Cervini ${ }^{4}$

\begin{abstract}
RESUMEN
El síndrome de Alagille (SA) se caracteriza por la presencia de colestasis crónica ocasionada por hipoplasia de las vías biliares; anomalías vertebrales, oculares y cardíacas, y fenotipo facial característico. Su herencia es autosómica dominante, causado en la mayoría de los casos por mutación del gen JAG1. Analizamos el caso de un niño de 3 años con diagnóstico de SA que, debido a las alteraciones lipídicas asociadas, presentó múltiples xantomas en la cara, el tronco y las extremidades, asociados a un prurito intenso.
\end{abstract}

El objetivo de la publicación es describir a un paciente con SA y múltiples lesiones cutáneas asociadas a un intenso prurito refractario. Destacamos la importancia de pesquisar las alteraciones del colesterol 0 una hepatopatía asociada en los pacientes con xantomas cutáneos. Palabras clave: colestasis, hipercolesterolemia, JAG1, síndrome de Alagille, xantomas.

Dermatol. Argent. 2020, 26 (3): 127-129

\section{ABSTRACT}

Alagille syndrome (ALGS) is characterized by the presence of chronic cholestasis caused by bile duct hypoplasia, vertebral, ophthalmic and cardiac abnormalities, as well as a characteristic facial phenotype. Its inheritance is autosomal dominant, most frequently caused by a mutation in the JAG1 gene. We present a 3-year-old boy with ALGS who presented with widespread pruritic xanthomas localized on the face, trunk, and limbs due to the associated hypercholesterolemia.
The aim of this publication is to describe a patient with ALGS and multiple skin lesions associated with intense refractory pruritus. We emphasize the importance of monitoring serum cholesterol levels and liver disease in patients with cutaneous xanthomas.

Key words: Alagille syndrome, cholestasis, hypercholesterolemia, JAG1, xanthomas.

Dermatol. Argent. 2020, 26 (3): 127-129
1 Médica Exbecaria, Servicio de Dermatología

2 Médica de Planta, Servicio de Dermatología

${ }^{3}$ Médica de Planta, Servicio de Anatomía Patológica

${ }^{4}$ Jefa del Servicio de Dermatología

Hospital Nacional de Pediatría Prof. Dr. Juan P. Garrahan, Ciudad

Autónoma de Buenos Aires, Argentina
Contacto del autor: María Candela Viada Peláez

E-mail: candeviada@hotmail.com

Fecha de trabajo recibido: $2 / 4 / 2020$

Fecha de trabajo aceptado: 6/9/2020

Conflicto de interés: los autores declaran que no existe conflicto de interés.

\section{CASO CLÍNICO}

Se presenta el caso de un niño de 3 años con diagnóstico clínico de síndrome de Alagille (SA) incompleto (enfermedad hepática colestásica crónica, defecto del tabique ventricular, embriotoxon posterior en el ojo derecho y facies peculiar) desde el año de vida, llevado a nuestro servicio por presentar múltiples lesiones cutáneas que aparecieron de forma progresiva durante el último año, asociadas a un prurito intenso, sin respuesta a los antihistamínicos instaurados.

En el examen físico el niño presentaba más de cien pápulas, induradas, color naranja amarillento, de su- perficie suave e indoloras, clínicamente compatibles con xantomas. Las lesiones afectaban la cara, el tronco y las extremidades, con compromiso palmoplantar y respetaban el cuero cabelludo, las mucosas y el área del pañal. El tamaño de estas pápulas oscilaba desde pocos milímetros hasta $3 \mathrm{~cm}$ y se agminaban de placas (Fotos 1 a 3). Además, se observó un tinte ictérico cutáneo generalizado asociado a una facies peculiar (enoftalmos, mentón pequeńo y orejas desplegadas) y hepatomegalia. El laboratorio solicitado informó colestasis (bilirrubina total 10,5 mg/dl, GOT 636 U/L, GPT 440 U/L, GGT 


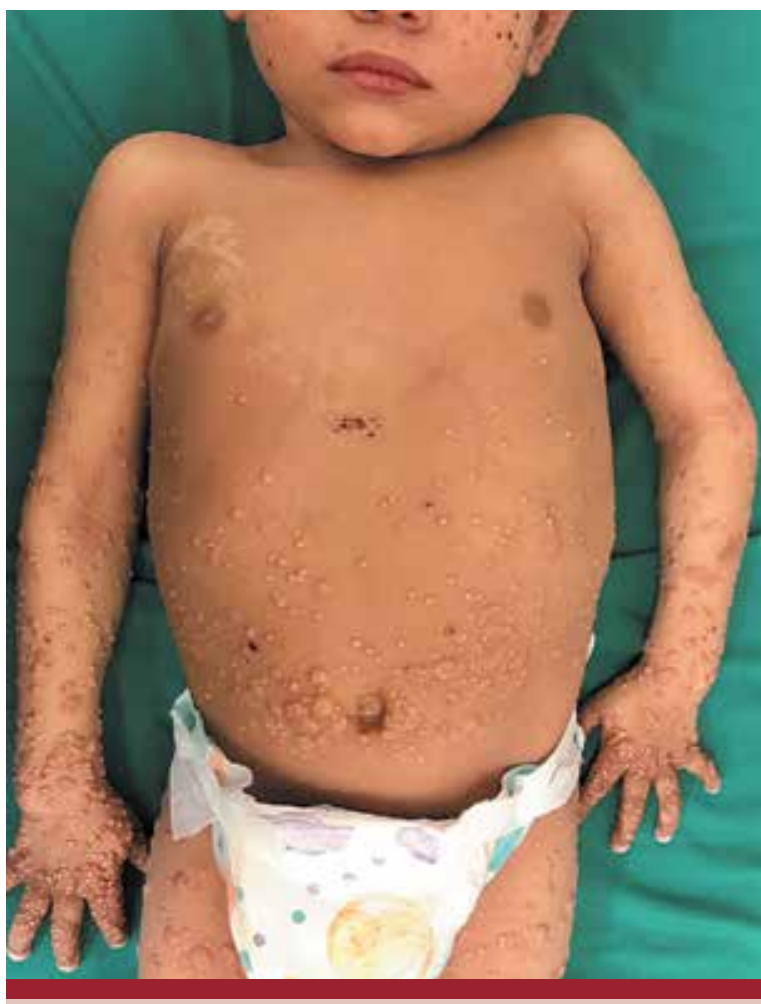

F0T0 1: Múltiples pápulas induradas, de coloración amarillenta, con distribución generalizada.

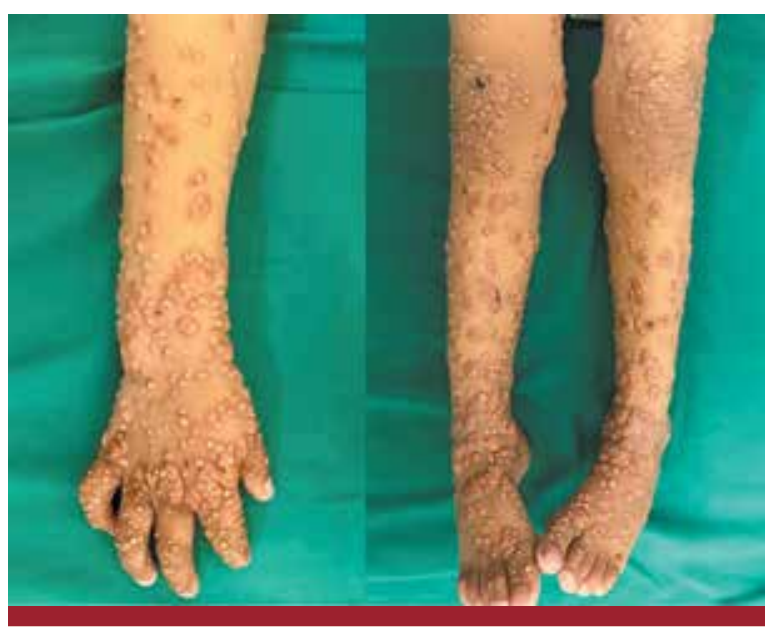

FOTO 2: Múltiples xantomas en las extremidades, costras secundarias a las excoriaciones.

$674 \mathrm{U} / \mathrm{L})$, hipercolesterolemia $(1636 \mathrm{mg} / \mathrm{dl})$ e hipertrigliceridemia $(610 \mathrm{mg} / \mathrm{dl})$. Ante la sospecha clínica de xantomas cutáneos, se realizó una biopsia de piel que reveló la presencia de un infiltrado dérmico difuso, compuesto principalmente por histiocitos xantomizados y, en menor medida, por linfocitos, lo que confirmó el diagnóstico presuntivo de xantomas (Foto 4). En conjunto con el servicio de hepatología se indicó continuar con antihistamínicos y ácido ursodesoxicólico, sin lograr la disminución del número ni del tamaño de los xantomas, como tampoco mejoría de la intensidad del prurito y de
FOTO 3: Xantomas con distribución generalizada: compromiso de la región facial, el pabellón auricular y los miembros superiores.

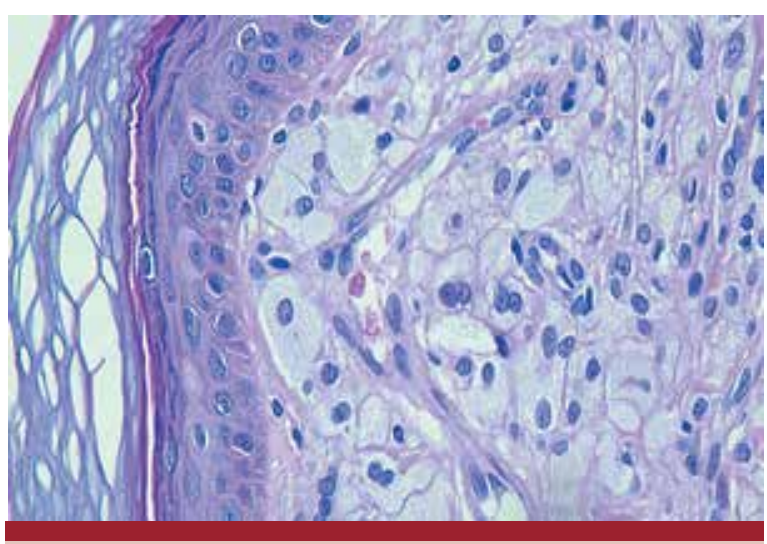

F0T0 4: Histiocitos xantomizados entremezclados con los haces de colágeno en la dermis (HyE, 10X).

las alteraciones del laboratorio. Debido a la gravedad de su hepatopatía, el paciente se encuentra en lista de espera para un trasplante hepático.

\section{COMENTARIOS}

El SA (tipo 1 OMIM \#118450/variante genética tipo 2 OMIM \#610205) es una enfermedad genética con afección multisistémica caracterizada por hipoplasia de las vías biliares asociada a otras malformaciones, como facies peculiar, y alteraciones cardiológicas, oftalmológicas y/o vertebrales ${ }^{1-3}$. Alagille lo describió por primera vez en $1962^{\text {citado por } 4}{ }^{4}$. La prevalencia actual es de 1 en 30.000 a 50.000 nacidos vivos (antes subesti- 
mada, ya que el diagnóstico de SA se basaba exclusivamente en la presencia de hepatopatía neonatal). Afecta a ambos sexos por igual ${ }^{1,2,5}$.

El síndrome es causado por mutaciones en los genes JAG1 o NOTCH2. La mayoría de los casos (97\%) son causados por la haploinsuficiencia del gen $J A G 1$ (SA de tipo I) en el cromosoma 20p12.2. Un pequeño porcentaje de pacientes presentan mutaciones en el gen $\mathrm{NOTCH} 2$ (SA de tipo II), ubicado en el cromosoma $1 \mathrm{p} 12.8^{1-3,5-7}$. Ambos genes codifican proteínas implicadas en la vía de señalización $\mathrm{NOTCH}$, las cuales cumplen un papel importante en la determinación del destino celular ${ }^{6,8,9}$. Su mutación genera una regulación negativa en los colangiocitos, hepatocitos y células mesenquimatosas y alteraciones en el desarrollo. Se hereda de forma autosómica dominante (30-50\%) y alrededor del 50-70\% son mutaciones de novo. Su expresividad fenotípica es variable?

El diagnóstico se basa en la presencia de cinco anomalías clínicas principales, denominados criterios clásicos: 1) colestasis crónica debida a la disminución del número de conductos biliares; 2) fenotipo facial característico (frente prominente, ojos hundidos con hipertelorismo, mentón puntiagudo, nariz recta y orejas grandes desplegadas); 3) anomalías cardiovasculares congénitas (estenosis de las arterias pulmonares y comunicación interventricular, entre otras); 4) defectos esqueléticos (principalmente vertebrales) y 5) anomalías oftalmológicas (la mayoría de las veces, embriotoxon posterior). Nuestro paciente presentó cuatro criterios clásicos (sin anomalías vertebrales). Otros hallazgos, considerados criterios menores, son: xantomas, retraso del crecimiento, retardo mental, pubertad tardía, alteraciones renales, anomalías óseas, insuficiencia pancreática, anomalías vasculares, hipogonadismo y cambios en el tono de la $\mathrm{voz}^{2,4-10}$. Los pacientes que tienen las cinco características clásicas se definen como SA completo, mientras que aquellos con cuatro características, como en el caso comentado, tienen la forma incompleta ${ }^{4,5}$.

\section{BIBLIOGRAFÍA}

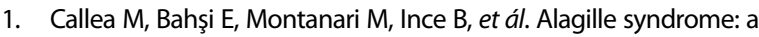
review. J Int Dent Med Res 2013;6:54-58.

2. Saleh M, Kamath BM, Chitayat D. Alagille syndrome: clinical perspectives. Appl Clin Genet 2016;9:75-82.

3. Turnpenny PD, Ellard S. Alagille syndrome: pathogenesis, diagnosis and management. Eur J Hum Genet 2012;20:251-257.

4. García MA, Ramonet M, Ciocca M, Cabrera $\mathrm{H}$, et ál. Alagille syndrome: cutaneous manifestations in 38 children. Pediatr Dermatol 2005;22:11-14.

5. Ruiz-Castillo M, Michel-Penichet F, Cervantes-Bustamante R, Zarate-Mondragón F, et ál. Síndrome de Alagille: informe de 12 casos en el Instituto Nacional de Pediatría. Rev Enfer Infec Pediatr 2007;21:13-17.

6. Ciocca M, Álvarez F. Síndrome de Alagille. Arch Argent Pediatr 2012;110:509-515.
Las lesiones cutáneas observadas en el SA son la ictericia, los xantomas y las excoriaciones debido al intenso prurito $^{2,4,10}$. Los xantomas suelen aparecer en el contexto de una hipercolesterolemia grave (superior a $500 \mathrm{mg} / \mathrm{dl}$ ) en relación con la colestasis crónica. Aunque la fisiopatología de esta afección no se conoce con exactitud, se ha propuesto que los lípidos séricos que se depositan en los tejidos son fagocitados por los macrófagos. Clínicamente, los xantomas son pápulas induradas, de coloración amarillenta, asintomáticas, de diferentes tamaños, que pueden aparecer en cualquier parte del cuerpo. Estas lesiones tienden a desaparecer luego de la reducción de los niveles séricos de colesterol o después del trasplante de hígado, es decir, cuando la colestasis mejora. Los xantomas deben diferenciarse de los moluscos contagiosos o de los xantogranulomas juveniles ${ }^{10}$. El estudio histopatológico de los xantomas describe múltiples células espumosas en la dermis entremezcladas con haces de colágeno.

El seguimiento de los pacientes con SA debe ser multidisciplinario debido al compromiso multisistémico que presentan ${ }^{5,6}$. La evaluación por distintos especialistas será necesaria, según la edad y las manifestaciones clínicas de cada paciente, para establecer el diagnóstico y el tratamiento oportunos ${ }^{5,9}$.

La colestasis, el prurito y los xantomas se han tratado con éxito con agentes coleréticos (como el ácido ursodesoxicólico) que estimulan el flujo biliar ${ }^{6}$. El trasplante hepático debe plantearse en los pacientes con colestasis crónica grave y prolongada, asociada a retraso del crecimiento, xantomatosis deformantes $y / o$ fibrosis portal progresiva ${ }^{6}$. El pronóstico a largo plazo depende de la gravedad de la enfermedad hepática y de las malformaciones asociadas ${ }^{1,5}$.

El motivo de esta comunicación fue describir a un niño con SA con una presentación excepcional de múltiples xantomas generalizados. Asimismo, destacar la importancia de detectar hepatopatías o alteraciones lipídicas ante la presencia de múltiples xantomas en la edad pediátrica.

7. Pearson $\mathrm{HJ}$, Mosser JL, Jacks SK. The triad of pruritus, xanthomas, and cholestasis: Two cases and a brief review of the literature. Pediatr Dermatol 2017;34:305-308.

8. Micaglio E, Andronache AA, Carrera P, Monasky MM, et ál. Novel JAG1 Deletion variant in patient with atypical Alagille syndrome. Int JMol Sci 2019;20:6247.

9. Spinner NB, Gilbert MA, Loomes KM, Krantz ID. Alagille syndrome. 2000 [Updated 2019 Dec 12]. En: Adam MP, Ardinger HH, Pagon RA, et ál., eds. GeneReviews ' [Consulta: agosto 2020].

10. Tempark T, Chatproedprai S, Wititsuwannakul J, Wananukul S. A 3-year-old girl with underlying Alagille syndrome, presenting with generalized whitish papules. Clin Exp Dermatol 2015;40:339-341. 